\title{
Micro-CT-based improvement of geometrical and mechanical controllability of selective laser melted Ti6Al4V porous structures
}

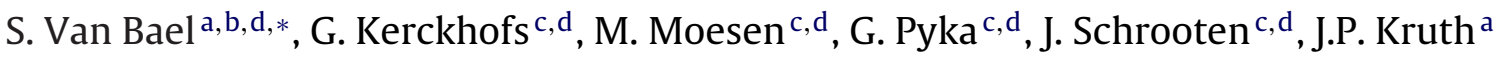 \\ a Department of Mechanical Engineering, Division of Production Engineering, Machine Design and Automation, Katholieke Universiteit Leuven, \\ Celestijnenlaan 300B, B-3001 Leuven, Belgium \\ ${ }^{\mathrm{b}}$ Department of Mechanical Engineering, Division of Biomechanics and Engineering Design, Katholieke Universiteit Leuven, Celestijnenlaan 300C, B-3001 Leuven, Belgium \\ ${ }^{\mathrm{c}}$ Department of Metallurgy and Materials Engineering, Katholieke Universiteit Leuven, Kasteelpark Arenberg 44, B-3001 Leuven, Belgium \\ ${ }^{\mathrm{d}}$ Prometheus, Division of Skeletal Tissue Engineering, Katholieke Universiteit Leuven, O\&N 1, Minderbroedersstraat 8A, B-3000 Leuven, Belgium
}

\section{A R T I C L E I N F O}

\section{Article history:}

Received 17 May 2011

Received in revised form 14 June 2011

Accepted 15 June 2011

Available online 22 June 2011

\section{Keywords:}

Powder metallurgy

Titanium alloys

Porous materials

Mechanical characterization

Tomography

\begin{abstract}
A B S T R A C T
Despite the fact that additive manufacturing (AM) techniques allow to manufacture complex porous parts with a controlled architecture, differences can occur between designed and as-produced morphological properties. Therefore this study aimed at optimizing the robustness and controllability of the production of porous Ti6Al4V structures using selective laser melting (SLM) by reducing the mismatch between designed and as-produced morphological and mechanical properties in two runs. In the first run, porous Ti6Al4V structures with different pore sizes were designed, manufactured by SLM, analyzed by microfocus X-ray computed tomography (micro-CT) image analysis and compared to the original design. The comparison was based on the following morphological parameters: pore size, strut thickness, porosity, surface area and structure volume. Integration of the mismatch between designed and measured properties into a second run enabled a decrease of the mismatch. For example, for the average pore size the mismatch decreased from $45 \%$ to $5 \%$. The demonstrated protocol is furthermore applicable to other 3D structures, properties and production techniques, powder metallurgy, titanium alloys, porous materials, mechanical characterization, tomography.
\end{abstract}

(c) 2011 Elsevier B.V. All rights reserved.

\section{Introduction}

Among the ubiquitous applications of porous metals (flame arresters, filters, shock absorbers) [1-9], certain applications impose stringent constraints on their porous morphology. For aerospace lightweight structures and tissue engineering (TE) scaffolds, the internal porous geometry is tailored to obtain desired geometrical, mechanical or fluid transport properties [10,11]. Because the obtained properties can still be highly sensitive to local or systematic variations in e.g. volume fraction or feature size and shape, their production requires a robust technique with high controllability and repeatability in terms of those parameters.

\footnotetext{
* Corresponding author at: Department of Mechanical Engineering, Division of Production Engineering, Machine Design and Automation, Katholieke Universiteit Leuven, Celestijnenlaan 300B, B-3001 Leuven, Belgium. Tel.: +3216 32 27 72; fax: +3216322987

E-mail addresses: Simon.Vanbael@mech.kuleuven.be (S. Van Bael) Greet.Kerckhofs@mtm.kuleuven.be (G. Kerckhofs), Maarten.Moesen@mtm.kuleuven.be (M. Moesen), Gregory.Pyka@mtm.kuleuven.be (G. Pyka), Jan.Schrooten@mtm.kuleuven.be (J. Schrooten), Jean-Pierre.Kruth@mech.kuleuven.be (J.P. Kruth).
}

Production techniques like foaming and powder metallurgy are limited in their ability to control the internal shape of porous structures, causing the repeatability of morphology and physical properties to be low $[12,13]$. Additive manufacturing (AM) techniques provide, due to the layer-wise building method and their direct link with a computed aided design (CAD) model, the ability to produce porous structures with controlled pore and strut dimensions. For example, Li et al. [14] investigated indirect production of porous implants with 3D fibre deposition, and produced structures with controlled and repeatable pore shape and pore size distribution. However, shrinkage after sintering caused the morphological parameters after production to significantly differ from the designed ones. Selective laser melting (SLM) and electron beam melting (EBM), both direct AM techniques, have been used to produce porous Ti6Al4V structures with repeatable morphological properties [4,5,15-22]. However, also for these production techniques significant differences between designed and as-produced pore morphologies were noticed. It is thus inherently difficult to produce customized porous structures matching closely the envisioned morphological and physical requirements.

The aim of this study is to optimize the robustness and controllability of the production of porous Ti6Al4V structures using 


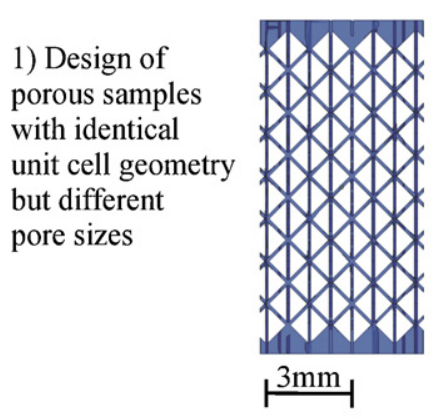

5) Compare designed
with measured
morphological
parameters
$\quad 700$

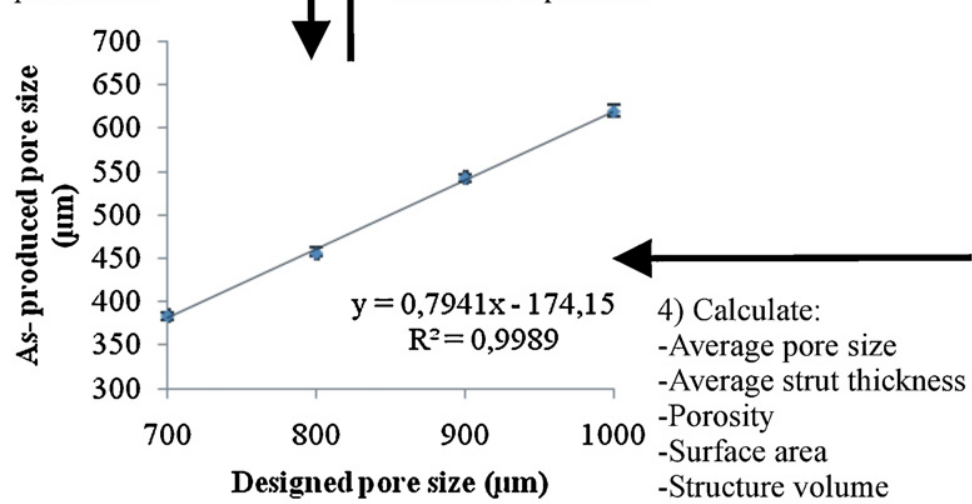

6) Adjust for mismatch

between designed and as-produced pore size using the experimentally obtained equations

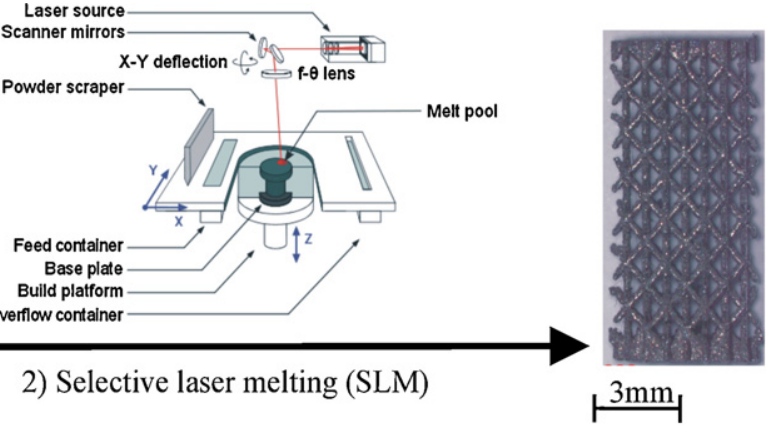

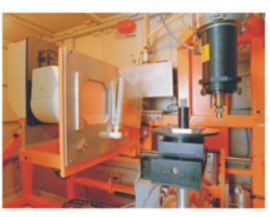

3) Micro-CT imaging
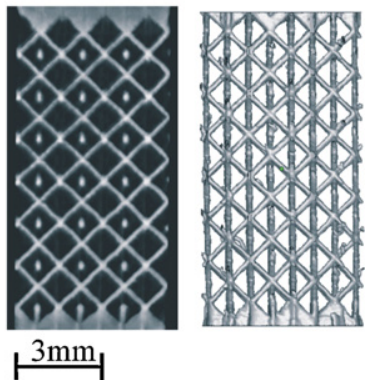

$3 \mathrm{~mm}_{1}$

Fig. 1. Schematic overview of the feedback loop between the design and the production including in-depth morphological characterization of the as-produced samples.

SLM by iteratively reducing the mismatch between designed and as-produced morphological and mechanical properties. For this purpose a feedback loop approach was performed two times, consisting of a design, production and in-depth morphological characterization step (Fig. 1). The first run, entitled the 'experimental' run, was needed to evaluate the initial controllability of the SLM process. After this experimental run, the mismatch between designed and as-produced morphology was used as input for the second run, named the 'production' run. The effectiveness of this compensation was verified at the end of the production run. For both runs the morphological properties were characterized by means of microfocus X-ray computed tomography (micro-CT), which for 3D and porous structures is a common measuring technique $[1,23-26]$.

\section{Materials and methods}

\subsection{Design and production of porous Ti6Al4V structures}

For the purpose of this study, 6 types of cylindrical samples (height $12 \mathrm{~mm}, \varnothing 6 \mathrm{~mm}$ ) consisting of a truss (i.e. framework of beams) were created using Magics software [Materialise NV, Haasrode, Belgium] based on the same unit cell (Fig. 2A) [22,27], but having different pore sizes. The designed pore size was the distance between two struts as given in Fig. 2C, ranging from 500 to $1000 \mu \mathrm{m}$ (Table 1). All 6 design geometries had a designed strut thickness (Fig. 2C) of $100 \mu \mathrm{m}$. Fig. 2B and D shows a typical STL-file of the design and a typical image of a produced sample with pore size $1000 \mu \mathrm{m}$.
Table 1 gives an overview of the 6 design geometries that were produced by SLM for 4 different assessments described in this study, namely (i) micro-CT image analysis, (ii) a repeatability study, (iii) compression testing and (iv) a specific design constraint.

In the experimental run, micro-CT image analysis and compression testing were performed on 5 randomly selected replicates of the 4 design geometries (Table 1 ).

The repeatability of the SLM process was evaluated using one particular design geometry (po 1000) that was manufactured on 5 different time points spread over 4 months.

In the production run, design geometries po 850 and po 500 were manufactured for validating the increased controllability. Note that the porosity of the former is within the range of the analyzed designs geometries in the experimental run, while the latter is outside this range.

All designs geometries were fabricated by a non-commercial, in-house developed SLM machine [28] using Ti6Al4V powder [Raymor Industries inc., Canada]. SLM is a layer-wise material addition technique that allows generating complex 3D parts by selectively melting successive layers of metal powder on top of each other, using the thermal energy supplied by a focused and computer controlled laser beam. The powder particles were spherical with a size distribution between 25 and $45 \mu \mathrm{m}$.

The SLM machine was equipped with a Yb:YAG fibre laser with beam spot size $80 \mu \mathrm{m}$ and a maximum power of $300 \mathrm{~W}$ on the powder bed. Because of high reactivity of Ti6Al4V to interstitial elements such as oxygen, nitrogen, carbon and hydrogen, the SLM process was carried out in a closed chamber flushed with argon gas to reduce the oxygen level below $0.1 \%$. SLM processing of the Ti6Al4V powder was conducted on a titanium base plate with a 


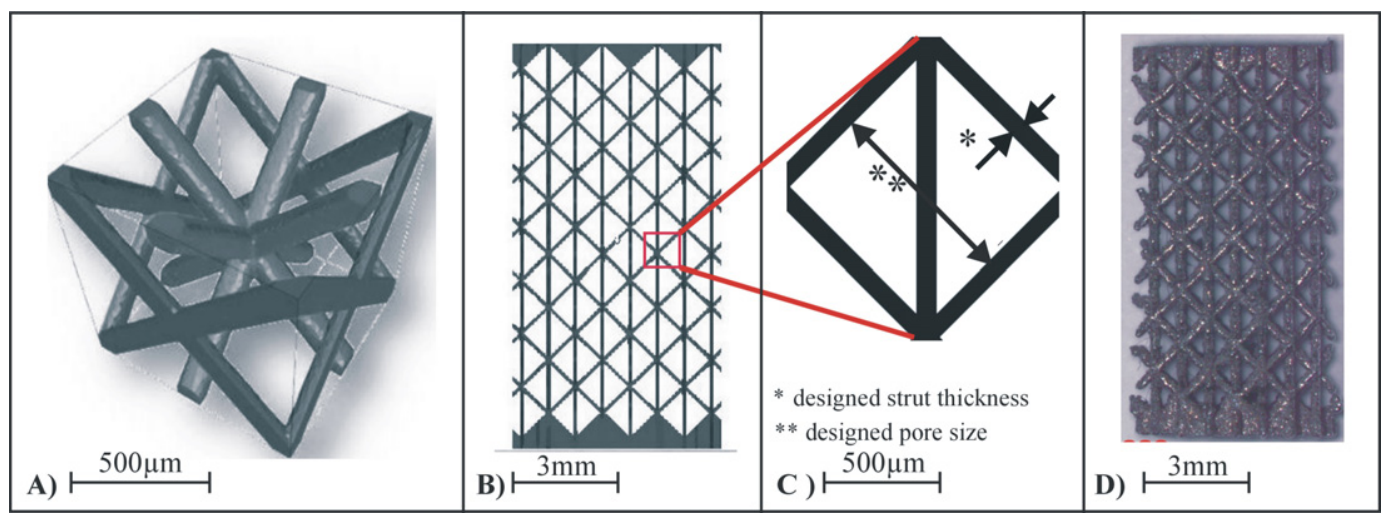

Fig. 2. (A) Unit cell of the designed porous structure. (B) STL design of a cylindrical porous structure with designed pore size $1000 \mu \mathrm{m}$, (C) directions of designed pore and strut thickness measurements and (D) a typical porous structure with designed pore size $1000 \mu \mathrm{m}$ manufactured by SLM.

Table 1

Overview of the 6 design geometries that were produced by SLM. $\sqrt{ }$ indicates the usage of the design in the corresponding part of this study.

\begin{tabular}{|c|c|c|c|c|c|c|}
\hline Design & Po 500 & Po 700 & Po 800 & Po 850 & Po 900 & Po 1000 \\
\hline Designed pore size $(\mu \mathrm{m})$ (Fig $2 \mathrm{~B})$ & 500 & 700 & 800 & 850 & 900 & 1000 \\
\hline Designed beam thickness ( $\mu \mathrm{m})$ (Fig 2B) & 100 & 100 & 100 & 100 & 100 & 100 \\
\hline Micro-CT image analysis $(n=5)$ & & $\sqrt{ }$ & $\sqrt{ }$ & & $\sqrt{ }$ & $\sqrt{ }$ \\
\hline Repeatability study $(n=5)(\#$ time $=5)$ & & & & & & $\sqrt{ }$ \\
\hline Compression testing $(n=5)$ & & $\sqrt{ }$ & $\sqrt{ }$ & & $\sqrt{ }$ & $\sqrt{ }$ \\
\hline Specific design constraint $(n=5)$ & $\sqrt{ }$ & & & $\sqrt{ }$ & & \\
\hline
\end{tabular}

laser power of $42 \mathrm{~W}$ and a scanning velocity of $260 \mathrm{~mm} / \mathrm{s}$. The powder layer thickness was $30 \mu \mathrm{m}$.

\subsection{Morphological characterization}

All 6 design geometries were scanned using a Philips HOMX 161 X-ray CT system with AEA tomahawk CT software [23]. During the acquisition, the manufactured sample was rotated over $187^{\circ}$ in steps of $0.5^{\circ}$. After each rotation, 32 images were acquired and the average radiograph was saved, resulting in a total of 374 radiographic images. These were reconstructed into cross-sectional images with a commercial software package [NRecon, Skyscan N.V., Kontich, Belgium]. The reconstructed micro-CT dataset had an isotropic voxel size of $(12.6 \mu \mathrm{m})^{3}$. This dataset was further analyzed using commercially available image analysis software [CTan, Skyscan N.V., Kontich, Belgium] and was additionally visualized with VGStudio Max 2.1 [Volume Graphics GMBH, Heidelberg, Germany].

Using the micro-CT image analysis the average strut thickness and pore size, porosity, surface area, structure volume and interconnectivity were calculated. Segmentation of the micro-CT images was based on the methodology described in Refs. [23,26].

\subsection{Mechanical characterization}

For compression testing of the SLM produced porous Ti6Al4V structures, an Instron universal test bench [Instron, Nordwood, U.S.], type 4505 , equipped with a load cell of $5 \mathrm{kN}$ and an extensometer was used at a compression rate of $0.2 \mathrm{~mm} / \mathrm{min}$. The stiffness, ultimate compressive strength and strain at maximum load were determined from the stress-strain curves. Since the curves did not show a distinct linear part in their stress-strain curve and its derivative did not show a plateau, the maximum slope of the stress-strain curve was regarded as the sample stiffness, in accordance with ASTM standards E111 and D695.

\subsection{Statistical analysis}

In this study, $p$-values were calculated using an un-paired student's $t$-test and considered significant when $p<0.01$.

One-way unpaired ANOVA was used to analyze intra-batch variations using Analyse-it.

\section{Results and discussion}

\subsection{Morphological characterization}

The key 3D morphological parameters acquired from the microCT analyses are porosity, interconnectivity, surface area, strut thickness and pore size distribution $[3,4]$. These were determined for 4 selected design geometries on 5 replicates (Fig. 3).

As a result from the increase in strut thickness with $112 \mu \mathrm{m}$ compared to the designed thickness (Figs. 3 and 4C), the porosity of the as-produced porous structures was systematically lower for all the design geometries compared to their design. In accordance, the structure volume and surface area increased significantly.

The increased strut thickness, and hence the mismatch for the different morphological properties is caused by three main reasons:

(1) The scan vectors, that describes the borders of a strut, were shifted $40 \mu \mathrm{m}$ inwards to compensate for the laser spot size $(80 \mu \mathrm{m})$. However, monitoring the melt pool when scanning with $42 \mathrm{~W}$ laser power and $260 \mathrm{~mm} / \mathrm{s}$ scan velocity showed a melt pool size of $\pm 180 \mu \mathrm{m}$ (Fig. 4A). It is also known that decreasing the laser power and increasing scan velocity can lead to a smaller melt pool. However changing these parameters would lead to an uncontrolled micro porosity in the struts which will decrease the controllability of the process $[28,29]$.

(2) The struts were built under a $45^{\circ}$ angle. Building at an angle will lead to an increasing strut thickness and waviness due to the staircase effect in additive manufactured parts (Fig. 4B) [30,31]. 


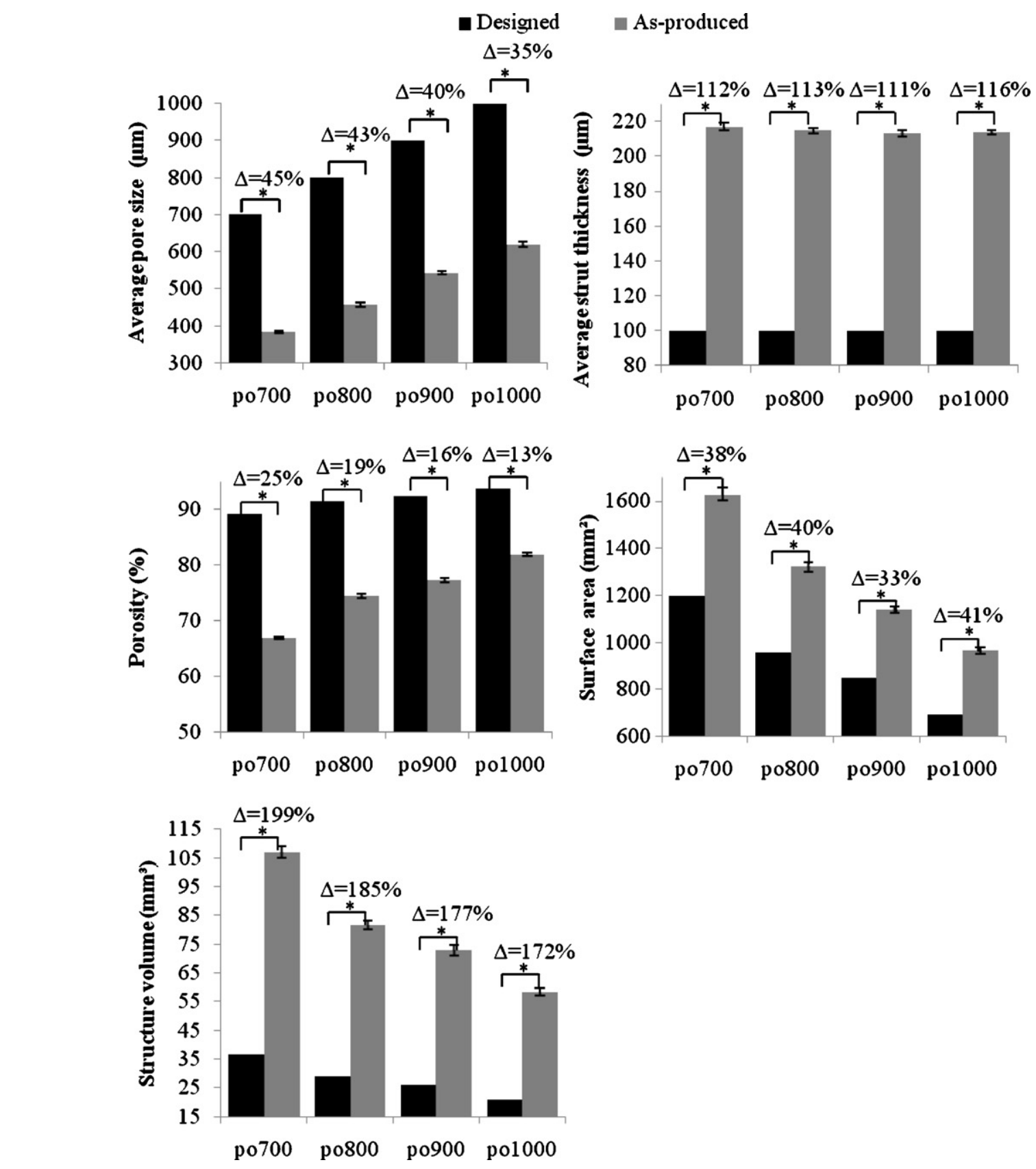

As-produced

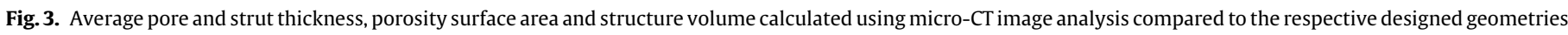

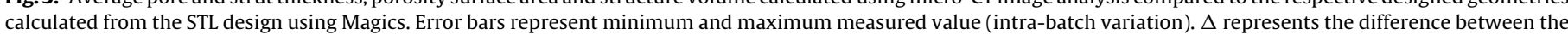
average as-produced and designed values. Statistical analysis; unpaired $t$-test $\left({ }^{*} p<0.01\right)$.

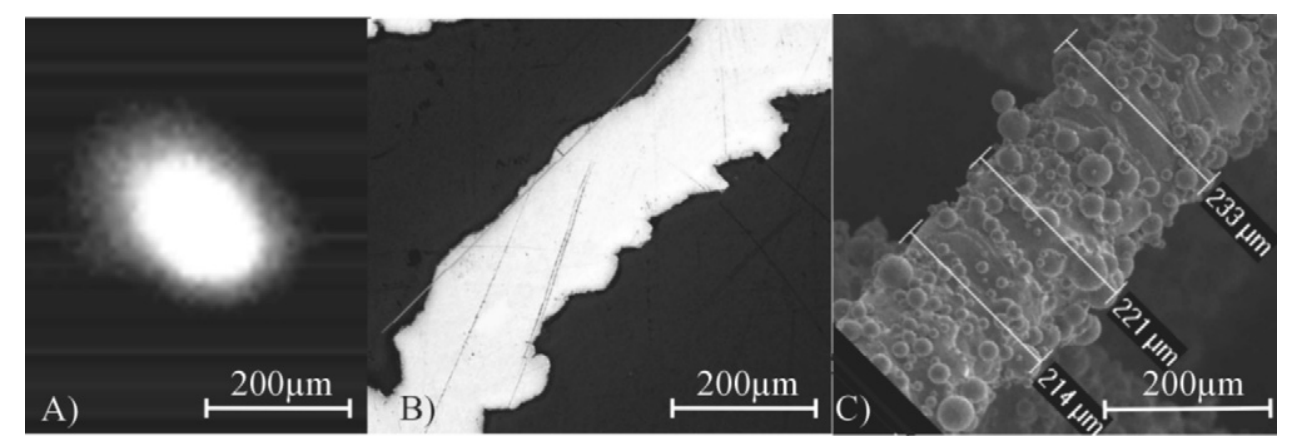

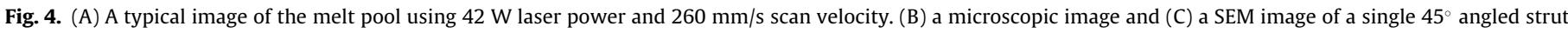
showing, the real strut size, a significant surface roughness and waviness caused by unmelted grains attached to the surface after production. 


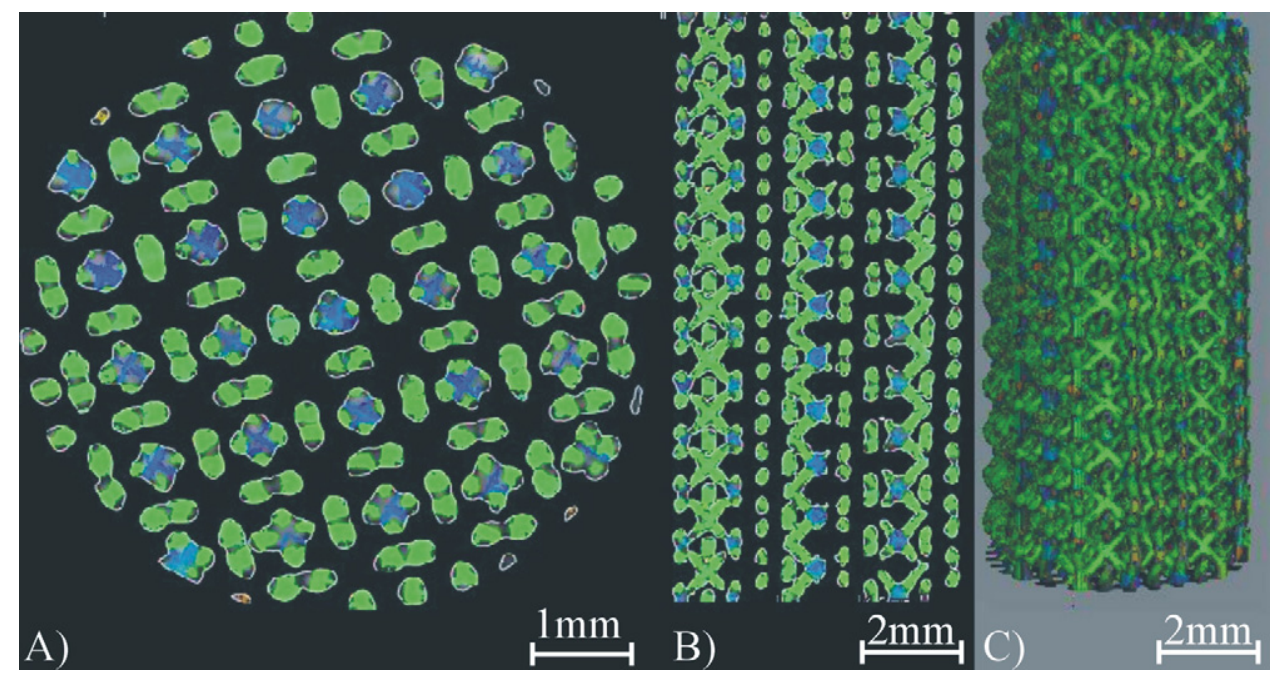

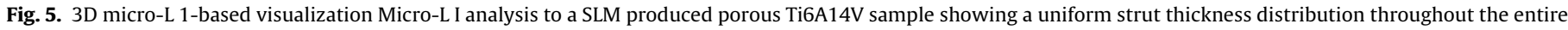

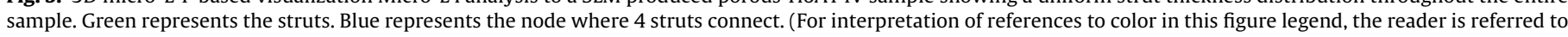
the web version of this article.)

(3) Angled struts are partially built on loose powder. A difference in heat transport between powder and solid material leads to powder particles sticking to the surface (Fig. 4C) [32,33].

Similar observations were made when producing porous Ti6Al4V structures using commercial AM machines. Indeed, Hollander et al. [16] reported in 2006 a mismatch of $150 \mu \mathrm{m}$ in the strut thickness between the design and the as-produced porous structures and thus decreased pore dimensions. Also Parthasarathy et al. [20] noticed a decrease in pore dimensions in electron beam melted porous structures compared to the design. Mullen et al. [32] investigated the effect of laser power, strut orientation and pore size on the resulted strut thickness. An increase in strut thickness with increasing laser power and strut angle and with decreasing pore size was noticed. However the above literature results were measured using 2D microscopy imaging limiting their measurements to the outer surface of the porous structure. Using 3D micro-CT instead of 2D microscopy imaging allowed us to calculate the as-produced porosity, surface area and structure volume, as well as the pore size and strut thickness distributions that are not limited to the outer surface of the structure. The latter distribution is visualized in Fig. 5 for an entire sample with designed pore size $1000 \mu \mathrm{m}$.

\subsection{Micro-CT image analysis to determine robustness and controllability of the SLM production technique}

Various research groups studied intra-batch geometries and found these to be low. They also found good correlations between designed and produced porosities, from which they concluded that they could adjust for the difference [15-17,34]. None of them however investigated inter-batch variations as function of time that may arise between structures with an identical design.

For this purpose, the repeatability of the SLM production technique was evaluated by micro-CT image analysis of po $1000(n=5)$ (Table 1) built on 5 different time periods. As shown in Fig. 6 the largest statistical difference in average pore size was only $14 \mu \mathrm{m}$, which from a practical point of view is still acceptable.

Therefore we consider the used SLM process as robust over time. As shown in Fig. 7 as-produced pore size, porosity, surface area and structure volume correlated well with the designed pore size. The empirical correlation functions, yielding $R^{2} \geq 0.95$, were considered suitable as input for a production run.

\subsection{Mechanical characterization}

The porosity and hence also the volume fraction ( $=1$ - porosity), are known to mainly determine the mechanical properties of porous structures $[15,16,18,34,35]$. Therefore the stiffness and ultimate compressive strength are measured on 5 replicates of 5 design variations of SLM produced porous Ti6Al4V structures (Fig. 8). The stiffness and ultimate compressive strength increased with decreasing porosity (decreasing pore dimensions and constant strut thickness) from respectively $296.73 \pm 17.22 \mathrm{MPa}$ to $1204.33 \pm 51.92 \mathrm{MPa}$ and from $9.69 \pm 0.15 \mathrm{MPa}$ to $33.64 \pm 0.83 \mathrm{MPa}$. The mechanical properties correlated well with the relative density $\left(R^{2}>0.97\right)$, which is promising in terms of controlling the mechanical properties. Moreover, the relatively high exponents $(>2)$ confirm that both stiffness and strength are very sensitive to the volume fraction [36].

This underlines the importance of increasing the controllability of the production method when a structure with controllable mechanical parameters is required. It can also be seen that numerical values of stiffness and ultimate strength using an analytical model similar to the foam model of Gibson and Ashby [36] were sys-

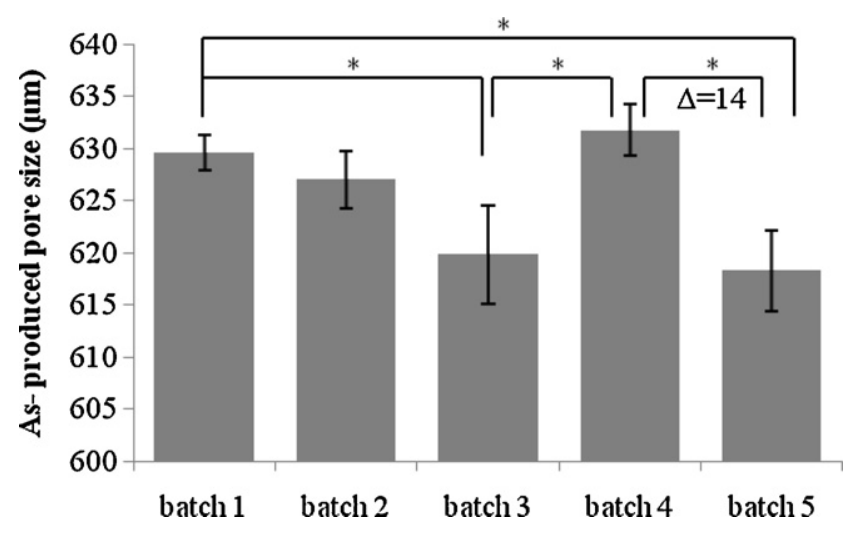

Fig. 6. The average pore size, determined using micro-CT image analysis, for 5 batch production series spread over multiple days, showing a small inter-batch variation. Error bars represent minimum and maximum measured value (intra-batch variation). A is the difference between maximum and minimum measured value. Statistical analysis: One-way unpaired ANOVA $\left({ }^{*} p<0.01\right)$. 

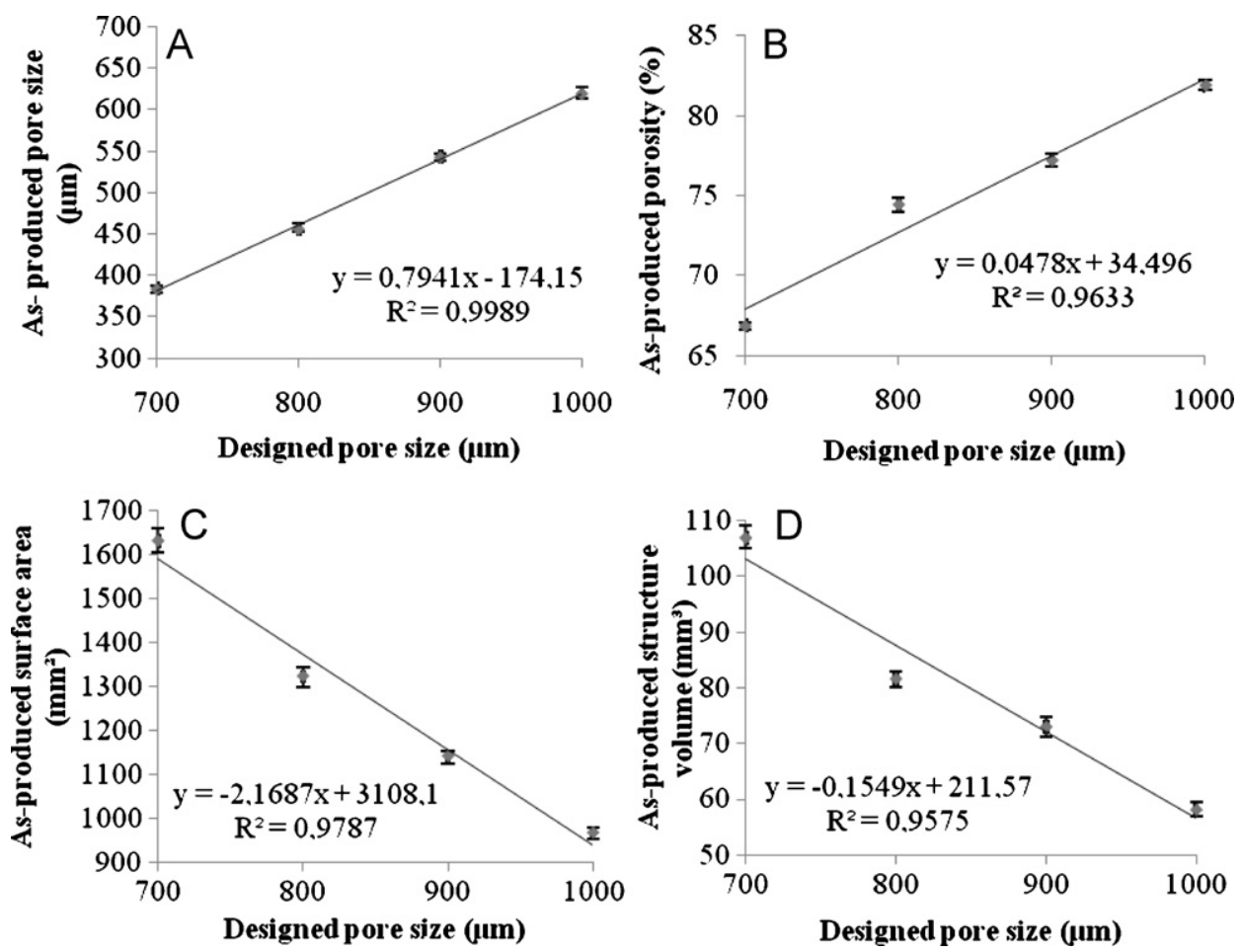

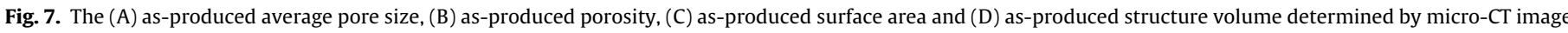

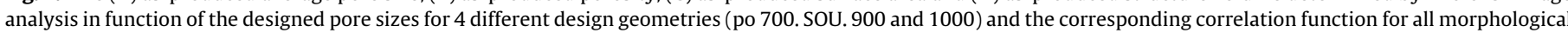
parameters. Error bars represent minimum and maximum measured values (intra-batch variation).

tematically higher than the experimentally obtained. Differences between experimental and numerical values were caused by three main reasons:

Residual stresses, which are inherent to SLM [37,38], occur in struts due to differences in cooling rate and shrinkage after melting. These stresses result in a lower compressive strength as they induce early strut failure by plastic yielding.

As shown in Fig. 4C powder particles remain attached to the strut surfaces. This additional volume of material contributes little to the mechanical strength. A post processing cleaning technique can remove these particles, resulting in smaller deviations [33].
Waviness and roughness of struts (Figs. 4B and C) will result in local heterogeneities and stress concentrations leading to a lower stiffness and lower compressive strength.

The above points complicate the accurate prediction of mechanical properties by means of analytical models and finite element analysis (FEA) [39,40]. However, as shown in Fig. 8, stiffness and compressive strength correlate well with volume fraction. Therefore the experimentally obtained correlations can be used for predicting those parameters. Note that the structure of the unit cell remains unchanged within the investigated range of volume fraction.
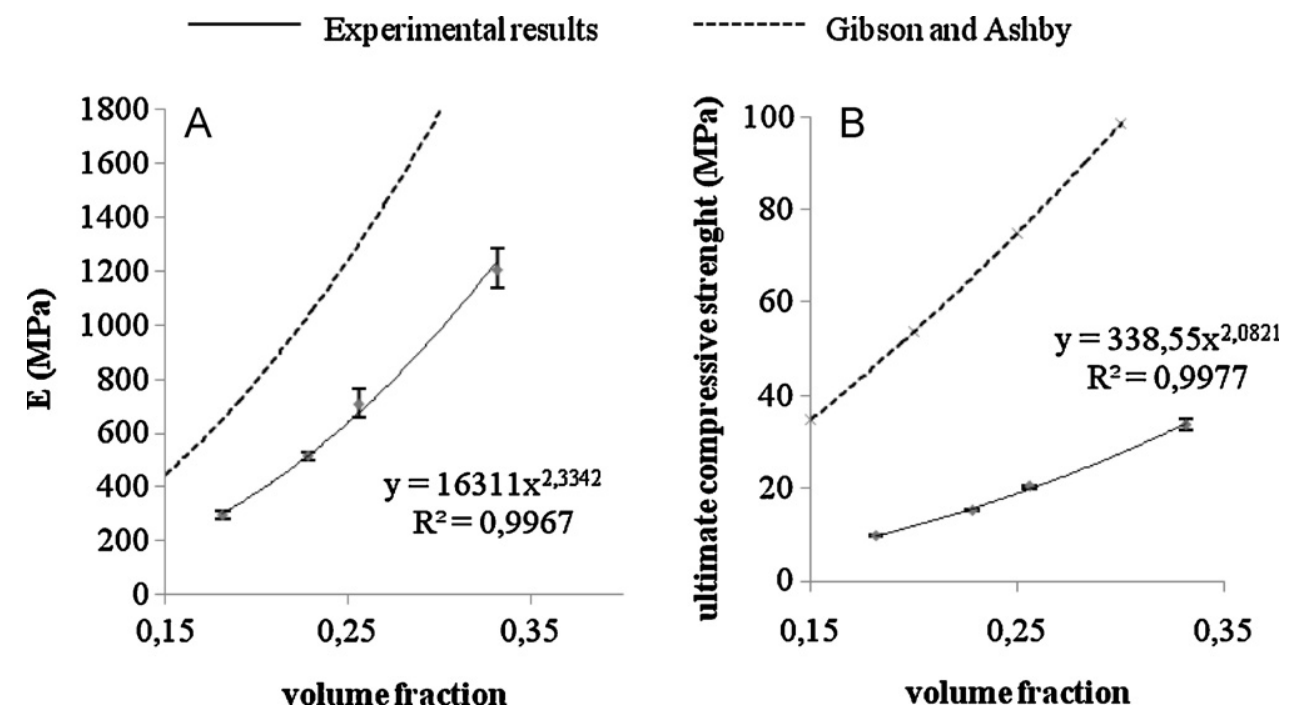

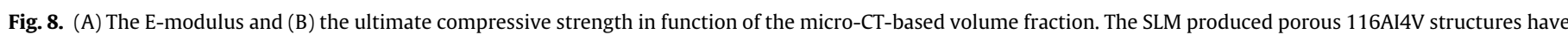

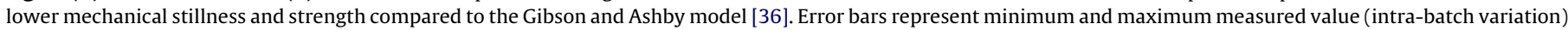




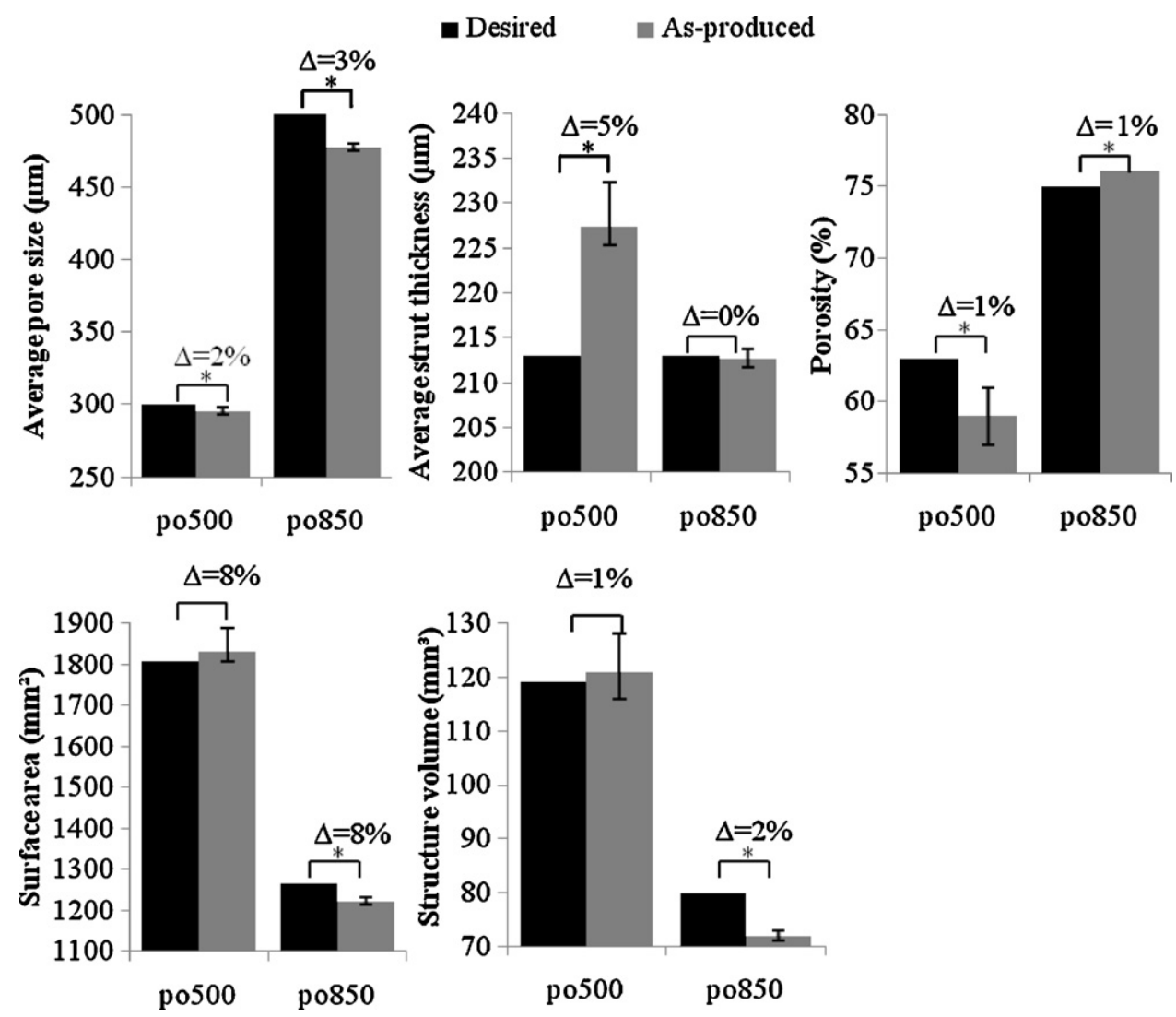

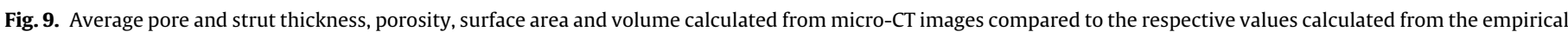

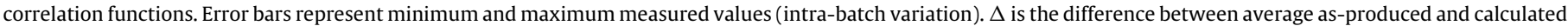
values. Statistical analysis unpaired $t$-test unpaired ( ${ }^{*}$ significantly different with $p<0.01$ ).

\subsection{Validating the protocol with a design constraint}

To validate whether the empirical correlation functions obtained in this study can be used for producing a porous structure with tailored morphological and mechanical properties, two porous structures with a desired average pore size of $300 \mu \mathrm{m}$ and $500 \mu \mathrm{m}$ were designed and manufactured with five replicates. Figs. 9 and 10 compare the morphological and mechanical properties predicted by previously discussed empirical correlation functions with those as acquired from micro-CT analysis and mechanical testing.

Statistically significant variations between micro-CT-based morphological parameters of the as-produced porous structures and the predicted values based on the empirical correlation functions are still present. However the differences are decreased tremendously compared to the structures analyzed in the experimental run.

The error bars in Fig. 9 represent the intra-batch variability. As this variability appeared to increase, average pore size where average pore size decreases, the production controllability for average pore sizes below $400 \mu \mathrm{m}$ will be lower.

The decrease in controllability, as described by Mullen et al. [32], is caused by diffusive heating of neighboring struts. Briefly, when the laser scans a strut, the surrounding powder will heat up due to thermal diffusion. This diffusive heat may in turn enlarge the melt pool of nearby built struts, causing them to be thicker.
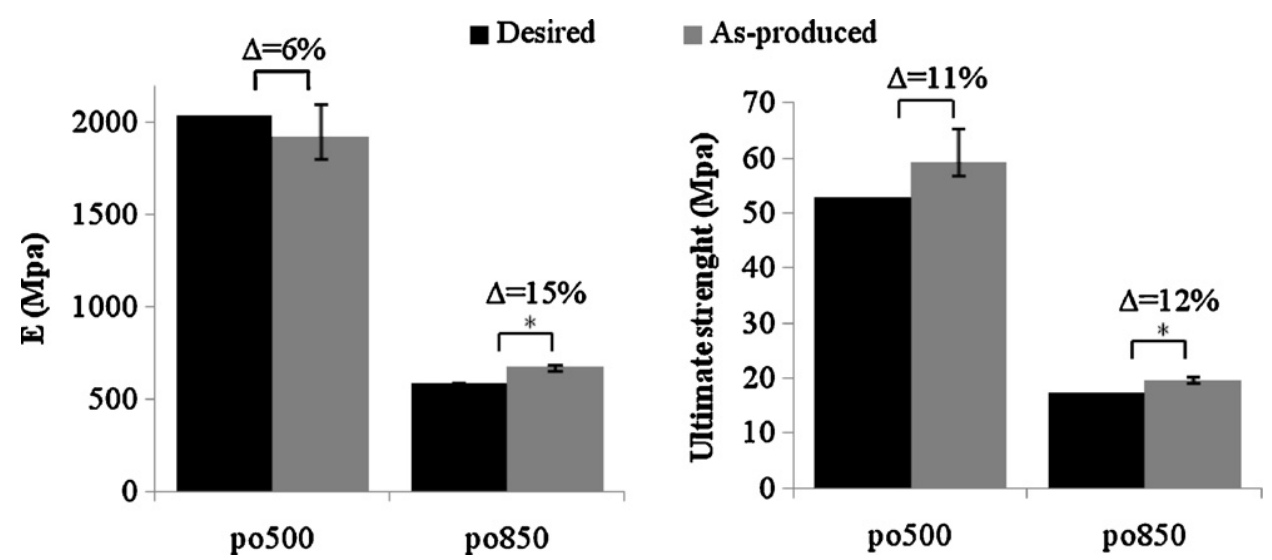

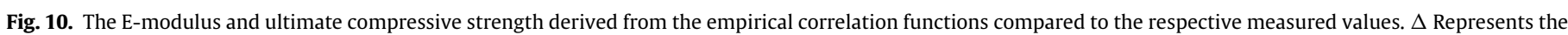
difference between the average as-produced and calculated values Statistical analysis unpaired $t$-test $\left({ }^{*} p<0.01\right)$. 
Lowering pore size further can eventually lead to closed pores [16,27].

Similar to the morphological parameters, variations between experimental obtained and predicted values were still present. The increased morphological intra-batch variation of the po 500 structure was reflected in the mechanical properties

The protocol, outlined in Fig. 1, consists of six steps and is essentially iterative. Our results showed that in practice two iterations (one experimental and one production) can be sufficient to drastically reduce the mismatch between designed and as-produced properties.

The design of samples in step (1) should vary the main geometrical parameters range to cover properties within a sufficiently wide region of interest. To identify these parameters and their ranges, predictive analytical and CAD models have proven to be helpful [39].

The production step (2) should avoid systematic production errors by using the appropriate technological constants, e.g. laser power, scan velocity and beam compensation offset since these constants are partially determining the resulted strut thickness. Nevertheless, some limited inter-batch and intra-batch variability remains unavoidable due to small fluctuations of process parameters like powder particle size and laser power. Other AM techniques like electron beam melting (EBM), 3D-printing (3DP) or fused deposition modeling (FDM) could replace SLM in this step, on condition that inter-batch and intra-batch variability of the chosen technique remains low. Micro-CT acquisition (3) and analysis (4) were essential in the first iteration for quantitatively determining 3D geometrical parameters. Relevant parameters such as the porosity (or relative density) and thickness distributions could be quantified sufficiently accurate given the current resolution. These parameters can be compared in step (5) and correlation functions can be established either empirically or with the aid of existing analytical and CAD models. In the latter case, the comparison may provide additional insight in the mechanical behavior of the porous structure as well as the limitations of the production technique. When the latter limitations cannot be resolved, we have shown that the correlation functions can be used within a certain range of validity, stipulated in step 1 , to nevertheless obtain the desired properties in a second production run.

While this protocol provides many benefits for the production of porous structures with tailored properties, there are certain limitations:

(1) The mismatch between design and as-produced (6) is an average value of all measured pores within one sample. Since the mismatch between design and as-produced is partially related to the unit cell, only porous structures with an identical unit cell throughout the whole structure can be controlled. However as it is possible to mark region of interests in the reconstructed micro-CT dataset, separate correlation functions for each unit cell could be established.

(2) A new experimental cycle, to obtain the correlation functions, is needed when one of the technical constants, strut angle or production method is changed.

(3) The spatial resolution of the micro-CT will limit the smallest detectable variations in the as-produced porous samples. Features smaller than the resolution, like surface roughness, will not be measured correctly. Therefore this protocol cannot be used if there is a need for a quantified surface roughness.

\section{Conclusions}

This work investigated a micro-CT-based protocol for increasing the controllability of porous structures produced by SLM. 3D
micro-CT image analysis showed a difference between designed and manufactured morphologic properties of the porous Ti6Al4V structures. A good repeatability of the SLM process over time was showed. Relevant empirical correlation functions were obtained for pore size, porosity, surface area and structure volume in function of the designed pore size, which allowed using the empirical correlation functions as prediction tools.

Mechanical properties correlated well with volume fraction $\left(R^{2}>0.97\right)$ following an analytical model. The obtained functions provided us with a tool for tailoring the mechanical properties without using FEA. The novelty of this protocol lies in the feedback of the experimentally obtained results to the design. Using the correlation functions for morphological and mechanical properties in a second run, we were able to produce porous structures in which the morphological and mechanical properties did meet the design constraints.

This protocol could be useful for applications where a good controllability in terms of morphological and mechanical parameters is needed. However future work is required to include different unit cell structures.

\section{Acknowledgements}

This work is funded by Quest (Quantitative Engineering of Skeletal Tissues) K.U.Leuven IDO 05/009 and is part of Prometheus, the Leuven Research \& Development Division of Skeletal Tissue Engineering of the Katholieke Universiteit Leuven: www.kuleuven.be/prometheus. Maarten Moesen and Greet Kerckhofs were funded by the agency for Innovation by Science and Technology in Flanders (IWT/OZM/080436 and IWT/OZM/090655).

\section{References}

[1] J. Banhart, Progress in Materials Science 46 (2001) 559-632.

[2] A. Ejlali, A. Ejlali, K. Hooman, H. Gurgenci, International Communications in Heat and Mass Transfer 36 (2009) 674-679.

[3] Y.H. Wang, J.P. Lin, Y.H. He, C.K. Zu, G.L. Chen, Journal of Alloys and Compounds 492 (2010) 213-218.

[4] I. Yadroitsev, I. Shishkovsky, P. Bertrand, I. Smurov, Applied Surface Science 255 (2009) 5523-5527.

[5] K. Alvarez, H. Nakajima, Materials 2 (2009) 790-832.

[6] Y.C. Chai, S. Truscello, S.V. Bael, F.P. Luyten, J. Vleugels, J. Schrooten, Acta Biomaterialia 7 (2011) 2310-2319.

[7] S. Das, S.J. Hollister, K.H.J. Buschow, W.C. Robert, C.F. Merton, I. Bernard, J.K. Edward, M. Subhash, V. Patrick, Encyclopedia of Materials: Science and Technology, Elsevier, Oxford, 2003, pp.1-7.

[8] S. McKown, Y.Shen, W.K. Brookes, C.J. Sutcliffe, W.J. Cantwell, G.S. Langdon, G.N. Nurick, M.D. Theobald, International Journal of Impact Engineering 35 (2008) 795-810.

[9] B. Stevens, Y. Yang, A. Mohandas, B. Stucker, K.T. Nguyen, Wiley Subscription Services Inc., A Wiley Company, 2008, pp. 573-582.

[10] S.J. Hollister, Nature Material 4 (2005) 518-524.

[11] D.W. Rosen, S.R. Johnston, M. Reed, H. Wang, Rapid Manufacturing Conference, Loughbourough, UK, 2006.

[12] K.F. Leong, C.M. Cheah, C.K. Chua, Biomaterials 24 (2003) 2363-2378.

[13] D.W. Hutmacher, M. Sittinger, M.V. Risbud, Trends in Biotechnology 22 (2004) 354-362.

[14] J.P. Li, J.R. de Wijn, C.A. Van Blitterswijk, K. de Groot, Biomaterials 27 (2006) 1223-1235.

[15] P. Heinl, L. Müller, C. Körner, R.F. Singer, F.A. Müller, Acta Biomaterialia 4 (2008) 1536-1544.

[16] D.A. Hollander, M. von Walter, T. Wirtz, R. Sellei, B. Schmidt-Rohlfing, O. Paar, H.-J. Erli, Biomaterials 27 (2006) 955-963.

[17] X. Li, C. Wang, W. Zhang, Y. Li, Materials Letters 63 (2009) 403-405.

[18] L.E. Murr, S.A. Quinones, S.M. Gaytan, M.I. Lopez, A. Rodela, E.Y. Martinez, D.H. Hernandez, E. Martinez, F. Medina, R.B. Wicker, Journal of the Mechanical Behavior of Biomedical Materials 2 (2009) 20-32.

[19] L.E. Murr, S.M. Gaytan, F. Medina, E. Martinez, J.L. Martinez, D.H. Hernandez, B.I. Machado, D.A. Ramirez, R.B. Wicker, Materials Science and Engineering: A 527 (2009) 1861-1868.

[20] J. Parthasarathy, B. Starly, S. Raman, A. Christensen, Journal of the Mechanical Behavior of Biomedical Materials 3 (2010) 249-259.

[21] G.E. Ryan, A.S. Pandit, D.P. Apatsidis, Biomaterials 29 (2008) 3625-3635. 
[22] S. Van Bael, G. Kerckhofs, M. Moesen, J.-P. Kruth, J. Schrooten, in: P. Bártolo (Ed.), International Conference on Tissue Engineering (ICTE2009), Bártolo, P., Leiria, Portugal, 2009.

[23] G. Kerckhofs, MTM, K.U. Leuven, Leuven, 2009.

[24] S.T. Ho, D.W. Hutmacher, Biomaterials 27 (2006) 1362-1376.

[25] J. Jones, R. Atwood, G. Poologasundarampillai, S. Yue, P. Lee, Journal of Materials Science: Materials in Medicine 20 (2009) 463-471.

[26] G. Kerckhofs, J. Schrooten, T. Van Cleynenbreugel, S.V. Lomov, M. Wevers, Review of Scientific Instruments 79 (2008) 013711-013719.

[27] S. Van Bael, B. Vandenbroucke, G. Kerckhofs, J. Schrooten, J. Kruth, 38th TMS Annual Meeting and Exhibition. TMS 2009, The Minerals, Metals \& Materials Society (TMS), San Francisco, USA, 2009.

[28] J. Van Vaerenbergh, Twente, Twente, Enschede, Netherland, 2008.

[29] L. Thijs, F. Verhaeghe, T. Craeghs, J.V. Humbeeck, J.-P. Kruth, Acta Materialia 58 (2010) 3303-3312.

[30] H.-S. Byun, K.H. Lee, Robotics and Computer-Integrated Manufacturing 22 (2006) 69-80.

[31] R.I. Campbell, M. Martorelli, H.S. Lee, Computer-Aided Design 34 (2002) 717-725.
[32] L. Mullen, R.C. Stamp, P. Fox, E. Jones, C. Ngo, C.J. Sutcliffe, Wiley Subscription Services, Inc., A Wiley Company, 2008, pp. 178-188.

[33] G. Pyka, G. Kerckhofs, S. Van Bael, M. Moesen, D. Loeckx, J. Schrooten, M. Wevers, European Conference for Non-Destructive Testing (ECNDT), Moscow, 2010.

[34] J.M. Williams, A. Adewunmi, R.M. Schek, C.L. Flanagan, P.H. Krebsbach, S.E. Feinberg, S.J. Hollister, S. Das, Biomaterials 26 (2005) 4817-4827.

[35] G. Ryan, P. McGarry, A. Pandit, D. Apatsidis, Wiley Subscription Services, Inc., A Wiley Company, 2009, pp. 894-906.

[36] L.J. Gibson, M.F. Ashby, Cellular Solids: Structure and Properties, Pergamon Press, 1997.

[37] A.H. Nickel, D.M. Barnett, F.B. Prinz, Materials Science and Engineering A 317 (2001) 59-64.

[38] J.P. Kruth, L. Froyen, J. Van Vaerenbergh, P. Mercelis, M. Rombouts, B. Lauwers, Journal of Materials Processing Technology 149 (2004) 616-622.

[39] M. Moesen, Department of Metallurgy and Materials Engineering, K.U. Leuven, Leuven, 2009, p. 266.

[40] M. Moesen, G. Kerckhofs, S. Van Bael, S.V. Lomov, I. Verpoest, J. Schrooten, ECCOMAS - International Conference on Tissue Engineering (ICTE), Leiria, Portugal, 2009. 